\title{
Correction to: Post-translational modification of OCT4 in breast cancer tumorigenesis
}

\author{
Yunhee Cho ${ }^{1,2} \cdot$ Hyeok Gu Kang ${ }^{1,2} \cdot$ Seok-Jun Kim ${ }^{3} \cdot$ Seul Lee $^{1,2} \cdot$ Sujin Jee ${ }^{1,2} \cdot$ Sung Gwe Ahn ${ }^{4}$ Min Jueng Kang ${ }^{5}$. \\ Joon Seon Song ${ }^{6,7} \cdot$ Joon-Yong Chung $\mathbb{D}^{7} \cdot$ Eugene C. Yi ${ }^{5} \cdot$ Kyung-Hee Chun ${ }^{1,2}$
}

Published online: 22 October 2019

(c) The Author(s), under exclusive licence to ADMC Associazione Differenziamento e Morte Cellulare 2019

\section{Correction to: Cell Death \& Differentiation}

$$
\text { https://doi.org/10.1038/s41418-018-0079-6 }
$$

The original PDF version of this article incorrectly showed the copyright holder to be 'ADMC Associazione
Differenziamento e Morte Cellulare 2018', when the correct copyright holder is 'The Authors 2018'. This has been corrected in the PDF version of the article.

Edited by S. Fulda

Kyung-Hee Chun

khchun@yuhs.ac

1 Department of Biochemistry and Molecular Biology, Yonsei University College of Medicine, 50 Yonsei-ro, Seodaemun-gu, Seoul 03722, Republic of Korea

2 Brain Korea 21 PLUS Project for Medical Science, Yonsei University College of Medicine, 50 Yonsei-ro, Seodaemun-gu, Seoul 03722, Republic of Korea

3 Department of Biomedical Science, College of Natural Science, Chosun University, 309 Pilmun-daero, Dong-gu, Gwangju 61452, Republic of Korea

4 Department of Surgery, Gangnam Severance Hospital, Yonsei University College of Medicine, 50 Yonsei-ro, Seodaemun-gu, Seoul 03722, Republic of Korea

5 Department of Molecular Medicine and Biopharmaceutical Sciences, School of Convergence Science and Technology and College of Medicine or College of Pharmacy, Seoul National University, Seoul, Republic of Korea

6 Department of Pathology, Asan Medical Center, University of Ulsan College of Medicine, Seoul 05505, Republic of Korea

7 Experimental Pathology Laboratory, Laboratory of Pathology, National Cancer Institute, National Institutes of Health, Bethesda, MD 20892, USA 